\title{
On the design of Spherical Gradient index lenses
}

\author{
J. C. Miñano*ab , D. Grabovičkića ${ }^{a}$ P. Benítez ${ }^{\text {ab }}$, J. C. González ${ }^{a}$, A. Santamaría ${ }^{a}$ \\ ${ }^{a}$ CEDINT, Universidad Politécnica de Madrid (UPM), Campus de Montegancedo 28223 Pozuelo, \\ Madrid, Spain; ${ }^{\mathrm{b}}$ LPI 2400 Lincoln Ave., Altadena, CA USA 91001
}

\begin{abstract}
Classical spherical gradient index (GRIN) lenses (such as Maxwell Fish Eye lens, Eaton lens, Luneburg lens, etc.) design procedure using the Abel integral equation is reviewed and reorganized. Each lens is fully defined by a function called the angle of flight which describes the ray deflection through the lens. The radial refractive index distribution is obtained by applying a linear integral transformation to the angle of flight. The interest of this formulation is in the linearity of the integral transformation which allows us to derive new solutions from linear combinations of known lenses. Beside the review of the classical GRIN designs, we present a numerical method for GRIN lenses defined by the Abel integral equation with fixed limits, which is an ill-posed problem.
\end{abstract}

Keywords: Geometrical optics, GRIN lenses, Mathematical methods

\section{INTRODUCTION}

Spherical symmetric lenses made of isotropic gradient index media have always called the attention of optical designers. Today a renewed interest on these lenses has come from the analysis of the perfect imaging in the Wave Optics. Recently Leonhardt has proved the super-resolution properties of the Maxwell Fish Eye lens (MFE). This means that the MFE can produce images with details below the classic Abbe diffraction limit [1]. Unlike the negative refractive index perfect imaging devices [2], the MFE is a GRIN lens made of materials with a positive, isotropic refractive index distribution.

In this paper, we recall first the design procedure developed by Luneburg [3][4], introducing the concept of angle of flight from which a function related to the refractive index distribution can be obtained by a linear integral transformation. This transformation allows us to derive old and new solutions for GRIN lenses just from linear combination of known lenses. In this way we will see, for instance, that the Eaton lens is just "twice" the Luneburg lens or that the MFE is the Eaton lens "plus" a homogeneous media lens.

Beside the analysis of the classical GRIN lenses (we will call them Type A GRIN lenses), which are defined by the Abel integral equation, we present a numerical method for other GRIN lenses defined by the Abel integral equation with fixed limits (we will call them Type B GRIN lenses). While in the case of the Type A GRIN lenses, the only rays that pass through all the lens refractive layers are the ones with zero skew invariant, in the case of Type B GRIN lenses this happens to all the rays. This makes a fundamental difference between the two types of the GRIN lenses. Type B GRIN lenses can not be solved using classical Luneburg approach.

Due to the spherical symmetry of the GRIN lenses, any ray trajectory is contained in a plane passing through the center of symmetry. This means, that we can restrict our discussion to ray trajectories in a plane defined by the polar coordinates $(\rho, \theta)$.

\section{CONFORMAL TRANSFORMATION}

In order to simplify the next explanations we are going to use a conformal transformation from the plane $(\rho, \theta)$ to the plane $(x, y)$ given by

$$
x=\ln (\rho) \quad y=\theta
$$

This is the conformal transformation defined by the natural logarithm complex analytical function (see for instance [3]). Since the transformation is conformal, the ray trajectories in the plane $(\rho, \theta)$ with a refractive index distribution $m(\rho)$ are transformed in curves of the plane $(x, y)$ which are also rays if this plane has the refractive index distribution given by 


$$
n(x)=\rho m(\rho)
$$

The circle $\rho=1$ is transformed in the straight line $x=0$. The points inside the circle $\rho<1$ are transformed in the half plane $x<0$, while the points $\rho>1$ are transformed in the half plane $x>0$.

For instance, with this transformation, the Maxwell Fish Eye distribution $m_{\mathrm{MFE}}(\rho)=2 /\left(1+\rho^{2}\right)$ transforms into the perfect image forming linear distribution $n_{C}(x)=1 / \cosh (x)$ [3].

\section{STATEMENT OF THE DESIGN PROBLEM}

We are going to consider the following design problem: Calculate the refractive index distribution $m(\rho)$ of a lens either in the range $0 \leq \rho \leq 1$ or in the range $\rho \geq 1$, such that the rays impinging in the lens flight an angle inside it given by a function $\theta(h)$, where $h$ is the skew invariant of the ray. Figure 1 shows the definition of the angle of flight for two different cases of gradient index lenses, when the gradient index is in $\rho<1$, and when it is outside the unit circle. Lenses with gradient index in $\rho \leq 1$ are usually Type A, while in the other case we can find both Type A and Type B.
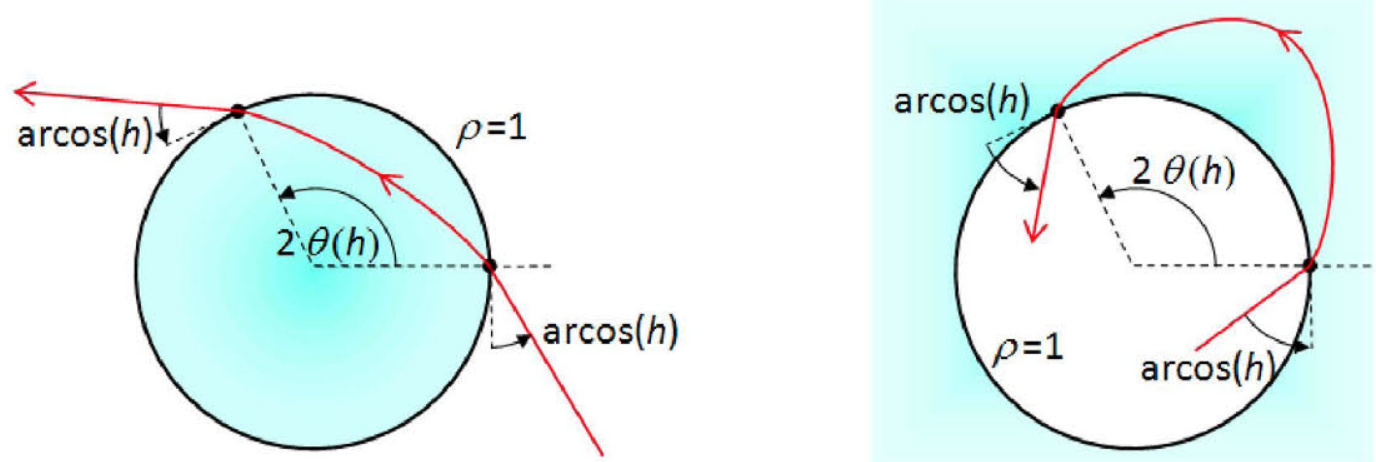

Figure 1 Angle of flight $\theta(h)$ definition when the gradient index is in $\rho<1$ (left) and when it is in $\rho>1$ (right). White media has refractive index 1 .

Figure 2 shows the same lenses form Figure 1, presented in the linear transformed space defined by Eq. (1). Now, the flight length is defined by the function $\mathrm{y}(q)$, where $p, q$ are direction cosines as shown in Figure 2.
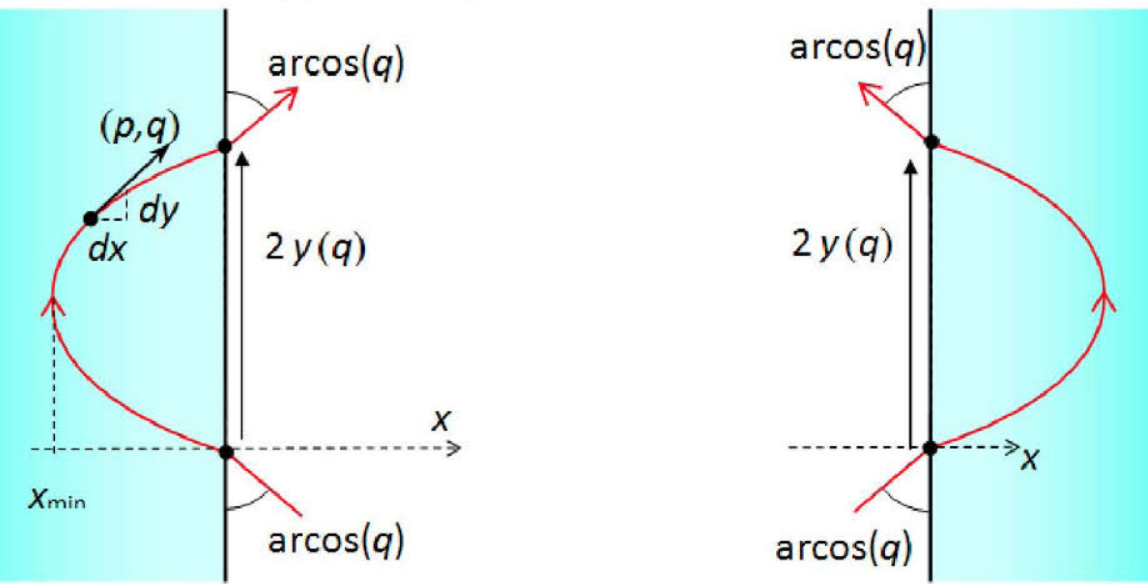

Figure 2 Length of flight $y(q)$ definition in the linear transformed spaces of the ones shown in Figure 1 


\section{TYPE A GRIN LENSES}

Consider a ray trajectory from Figure 2. The elemental increment of the coordinate $y$ is given as

$$
d y=\frac{q}{p(x)} d x=\operatorname{sign}(n) \frac{q}{\sqrt{n^{2}(x)-q^{2}}} d x
$$

We are going to assume that the refractive index distribution is such that $\mathrm{d}\left[n^{2}(x)\right] / \mathrm{dx} \geq 0$, for $\mathrm{x} \leq 0$, and that the $x$ coordinate of rays travelling find a minimum $x_{\min }$ beyond which they can't pass. By integrating the increment along the ray path inside the lens (for $x<0$ ) one gets

$$
\begin{aligned}
& \int_{0}^{y(q)} d y=\int_{x_{\min }(q)}^{0} \operatorname{sign}(n) \frac{q}{\sqrt{n^{2}(x)-q^{2}}} d x \\
& y(q)=q \int_{x_{\min }(q)}^{0} \frac{\operatorname{sign}(n)}{\sqrt{n^{2}(x)-q^{2}}} d x
\end{aligned}
$$

Note that $n^{2}\left(x_{\min }\right)=q^{2}$. Let's define the vraibales $s, t$ and $\chi$ as

$$
\begin{aligned}
& n^{2}=1-t, \quad \operatorname{sign}(n) d x=d \chi=\dot{\chi} d t \\
& q^{2}=1-s
\end{aligned}
$$

Note that when $\operatorname{sign}(n)=1$ every where (positive refractive index everywhere) then $\chi=x$ plus an additive constant. When there is a region with negative refractive index, then $d \chi=-d x$. We are going to normalize the refractive index distribution to the one corresponding to $x=0, n(0)$ or more precisely to $n\left(0^{-}\right)$defined as

$$
n\left(0^{-}\right)=\lim _{\substack{x \rightarrow 0 \\ x<0}} n(x)
$$

Then, we can state that when $x \rightarrow 0^{-}, n^{2} \rightarrow 1$ and so $t \rightarrow 0$, and the integral of Eq. (4) can be written

$$
f(s)=\int_{0}^{s} \frac{\dot{\chi}}{\sqrt{s-t}} d t
$$

where

$$
f(s)=-\frac{y(\sqrt{1-s})}{\sqrt{1-s}}
$$

Eq. (7) is a Fredholm integral equation of the first kind where $\dot{x}$ is the unknown function and $y(q)=y\left((1-s)^{1 / 2}\right)$ is known [5][6]. This integral equation is usually called Abel integral equation. Luneburg was the first to notice that this kind of problems can be stated as an Abel integral equation. The integral at the right hand of Eq. (7) is proportional to the fractional integral operator of order $1 / 2$ (noted by $I^{1 / 2}$ ) applied on the function $\chi$. This operator is defined as (this is the Riemann fractional integral, which is one of many possible definitions of fractional integral) [6].

$$
I^{1 / 2}[x(t)]=\frac{1}{\sqrt{\pi}} \int_{0}^{t} \frac{x(\tau)}{\sqrt{t-\tau}} d \tau
$$

Fractional integral operators $I^{\alpha}$ fulfill the semigroup property $I^{\alpha} I^{\beta}=I^{\alpha+\beta}$. This means, for instance, that applying twice the fractional integral operator $I^{1 / 2}$ to the function $x(t)$ we get $I[x(t)]$ which is simply the integral of $x(t)$

$$
I^{1 / 2}\left[I^{1 / 2}[x(t)]\right]=I[x(t)]=\int_{0}^{t} x(\tau) d \tau
$$

Fractional derivatives are defined in a similar way as fractional integral. There is not a unique definition. The Caputo fractional derivative is the most adapted to our problem. Using Caputo's definition we can write Eq. (7) as 


$$
\frac{f(s)}{\sqrt{\pi}}=D^{1 / 2}(\chi(s))
$$

Eq. (10) provides the key to solve the Abel integral. Applying again the fractional integral operator of order $1 / 2, I^{1 / 2}$ on both sides of Eq. (7) we get

$$
\frac{1}{\pi} \int_{0}^{t} \frac{f(s)}{\sqrt{t-s}} d s=\int_{0}^{t} \dot{\chi} d t=\chi(t)-\chi(0)
$$

Let's set $\chi(0)=0$. Then

$$
\begin{gathered}
\chi(t)=\frac{1}{\pi} \int_{0}^{t} \frac{f(s)}{\sqrt{t-s}} d s=-\frac{1}{\pi} \int_{0}^{t} \frac{y(\sqrt{1-s})}{\sqrt{1-s} \sqrt{t-s}} d s \\
\chi(t)=\frac{2}{\pi} \int_{1}^{\sqrt{1-t}} \frac{y(q)}{\sqrt{t-1+q^{2}}} d q \\
\chi=-\frac{2}{\pi} \int_{|n|}^{1} \frac{y(q)}{\sqrt{q^{2}-n^{2}}} d q
\end{gathered}
$$

Assuming that we can evaluate Eq. (15) then we can plot $\chi$ versus $n^{2}$. Because we have set $\chi(t=0)=0$, then $\chi\left(n^{2}=1\right)=0$. We have assumed that $n^{2}(x)$ is a non decreasing function in the domain $x<0$. We can also plot $\chi$ versus $n$, by assigning a negative refractive index when the $d \chi / d\left(n^{2}\right)<0$. Since $d \chi=d x$ when the refractive index at $x$ is positive and $d \chi=-d x$ in the opposite case, we can now plot $\chi$ versus $x$, and finally plot $n(x)$. Hence, the calculation of refractive index distribution for a GRIN lens is done by the following steps:

1. Start with $\theta=f_{1}(h)$. Note that the same function with different variables is the input data for the linear problem: $y=f_{1}(q)$. We are assuming that $n^{2}(x)$ is a non decreasing function in the domain $x<0$, i.e., $|n(x)| \leq n\left(0^{-}\right)$which is set to 1 by definition. Then there is no ray with $|q|>1$ that can enter into the half plane $x<0$. Consequently the function $y=f_{1}(q)$ is only defined in the range $|q| \leq 1$. Moreover, the function $y=f_{1}(q)$ must be odd $\left(f_{1}(-q)=\right.$ $\left.-f_{1}(q)\right)$ thus we only need to know it in the range $0 \leq q \leq 1$.

2. Calculate $\chi=f_{2}\left(n^{2}\right)$ with Eq. (15).

3. Calculate $\chi=f_{3}(n)$ by assigning a negative refractive index where the derivative $d f_{2} / d n^{2}$ is negative.

4. Calculate $\chi=f_{4}(x)$ with the equations $d \chi=d x$ (positive $n$ ) and $d \chi=-d x$ (negative $n$ ) with the contour condition $\chi=0$ when $x=0$.

5. Calculate $n=n(x)$ with $\chi=f_{3}(n)$ and $\chi=f_{4}(x)$.

6. Use Eq. (1) and (2) to calculate $m(\rho)$.

\subsection{Some examples for Type A GRIN lenses}

Table 1 shows some examples of Type A GRIN lenses.

Table 1 Examples of Type A GRIN lenses

\begin{tabular}{|l|l|l|}
\hline 1. & $\theta(h)=1 / 2(\pi-\arcsin (h))$, then $y(q)=1 / 2(\pi-\arcsin (q))$ \\
2. & $\chi=1 / 2 \ln \left[1-\left(1-n^{2}\right)^{1 / 2}\right]$ \\
3. & $d \chi / d\left(n^{2}\right)>0$ for $n^{2}<1$. Then there is no negative & \\
& index region
\end{tabular}




\begin{tabular}{|c|c|c|}
\hline 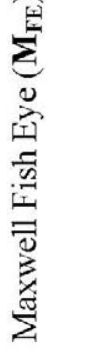 & $\begin{array}{ll}\text { 1. } & \theta(h)=\pi / 2, \text { and so } y(q)=\pi / 2 . \\
\text { 2. } & \chi=\ln \left[1-\left(1-n^{2}\right)^{1 / 2}\right]-\ln (n)=-\operatorname{Arg} \operatorname{Ch}(1 / n) \\
\text { 3. } & d \chi / d\left(n^{2}\right)>0 \text { for } n^{2}<1 . \text { Then there is no negative } \\
& \text { index region } \\
\text { 4. } & \chi=x \\
\text { 5. } & n(x)=2 /\left(\mathrm{e}^{-x}+\mathrm{e}^{x}\right) \\
\text { 6. } & m(\rho)=2 /\left(1+\rho^{2}\right)\end{array}$ & \\
\hline 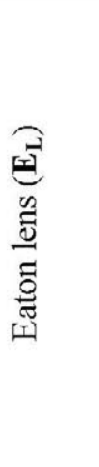 & $\begin{array}{l}\text { 1. } \quad \theta(h)=\pi-\arcsin (h), \text { then } y(q)=\pi-\arcsin (q) \text {. Since } \\
\text { this is twice the angle of flight of the Luneburg lens } \\
\text { and the fractional integral transform is linear, the } \\
\text { corresponding } \chi=\mathrm{f}_{2}\left(n^{2}\right) \text { must be twice that of the } \\
\text { Luneburg lens, i.e, } \mathbf{E}_{\mathbf{L}}=2 \mathbf{L}_{\mathbf{L}} \text {. } \\
\text { 2. } \chi=\ln \left\{1-\left(1-n^{2}\right)^{1 / 2}\right\} \\
\text { 3. } d \chi / d\left(n^{2}\right)>0 \text { for } n^{2}<1 . \text { Then there is no negative } \\
\text { index region } \\
\text { 4. } \chi=x . \\
\text { 5. } \\
\text { 6. } \\
m(x)=\left(2 \mathrm{e}^{x}-\mathrm{e}^{2 x}\right)^{1 / 2} . \\
m(\rho)=(2 / \rho-1)^{1 / 2} .\end{array}$ & \\
\hline 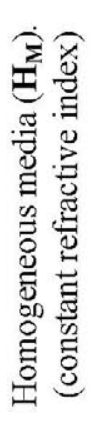 & $\begin{array}{l}\text { 1. } \theta(h)=\arccos (h) \text {, and so } y(q)=\arccos (q) \text {. This can } \\
\text { also be written as } y(q)=\pi / 2-\arcsin (q) \text {, i.e. the } \\
\text { difference of the angles of flight of the } \mathbf{E}_{\mathbf{L}} \text { and } \mathbf{M}_{\mathbf{F E}} \\
\text { We write } \mathbf{H}_{\mathbf{M}}=\mathbf{E}_{\mathbf{L}}-\mathbf{M}_{\mathbf{F E}} \text {. } \\
\text { 2. } \chi=\ln \left[1-\left(1-n^{2}\right)^{1 / 2}\right]+\ln (n)-\ln \left[1+\left(1-n^{2}\right)^{1 / 2}\right]=1 / 2 \ln \left(n^{2}\right) \\
\text { 3. } d \chi / d\left(n^{2}\right)>0 \text { for } n^{2}<1 \text {. Then there is no negative } \\
\text { index region } \\
\text { 4. } \chi=x \text {. } \\
\text { 5. } n(x)=\mathrm{e}^{x} . \\
\text { 6. } m(\rho)=1 .\end{array}$ & 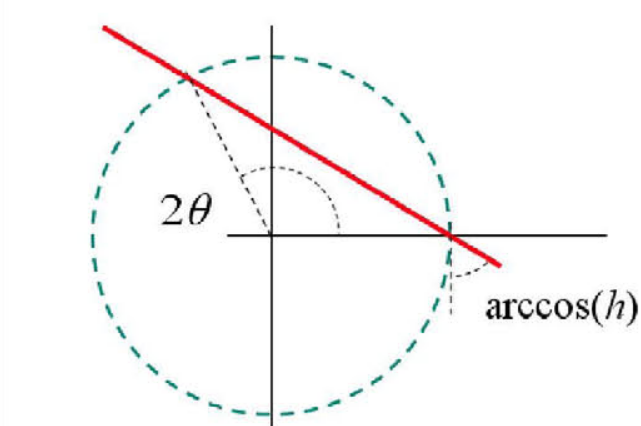 \\
\hline $\begin{array}{l}\sum_{0}^{3} \\
\frac{0}{0} \\
\sum\end{array}$ & $\begin{array}{l}\text { 1. This is a lens with gradient index in } \rho>1 \text {, whose } \\
\text { angle of flight is complementary to that of Eaton, } \\
\text { so } \theta(h)=\arcsin (h), \text { then } y(q)=\arcsin (q) . \quad \mathbf{M}_{\mathbf{L}}= \\
2\left(\mathbf{M}_{\mathrm{FE}}-\mathbf{L}_{\mathbf{L}}\right) . \text { Eq. }(15) \text { applies with a change of sign. } \\
\text { 2. } \chi=\ln \left[1+\left(1-n^{2}\right)^{1 / 2}\right] \\
\text { 3. } d \chi / d\left(n^{2}\right)<0 \text { for } n^{2}<1 \text {. Then there is no negative } \\
\text { index region } \\
\text { 4. } \chi=x . \\
\text { 5. } n(x)=\left(2 \mathrm{e}^{x}-\mathrm{e}^{2 x}\right)^{1 / 2} . \\
\text { 6. } m(\rho)=(2 / \rho-1)^{1 / 2} \text { in } \rho>1\end{array}$ & \\
\hline
\end{tabular}




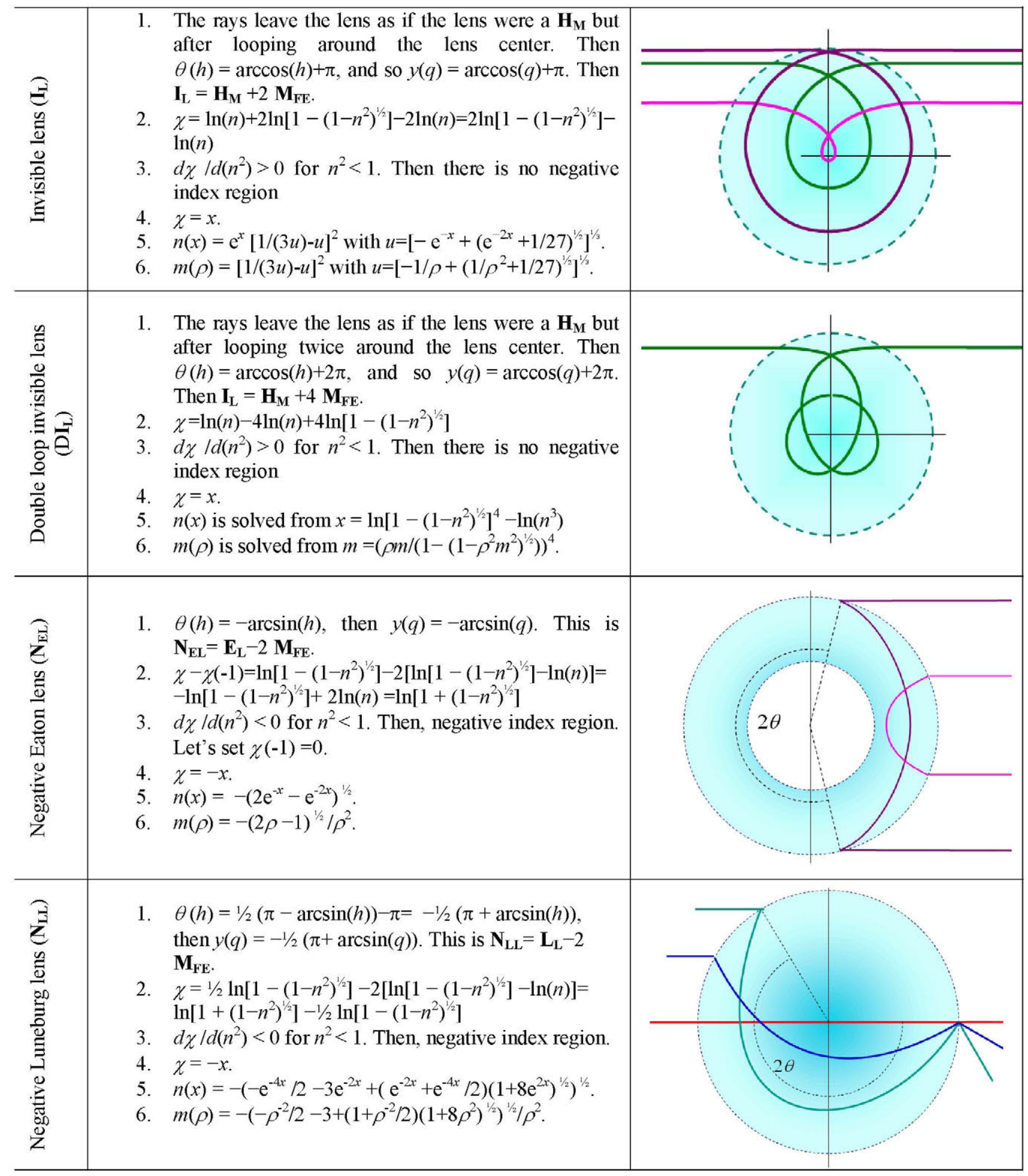




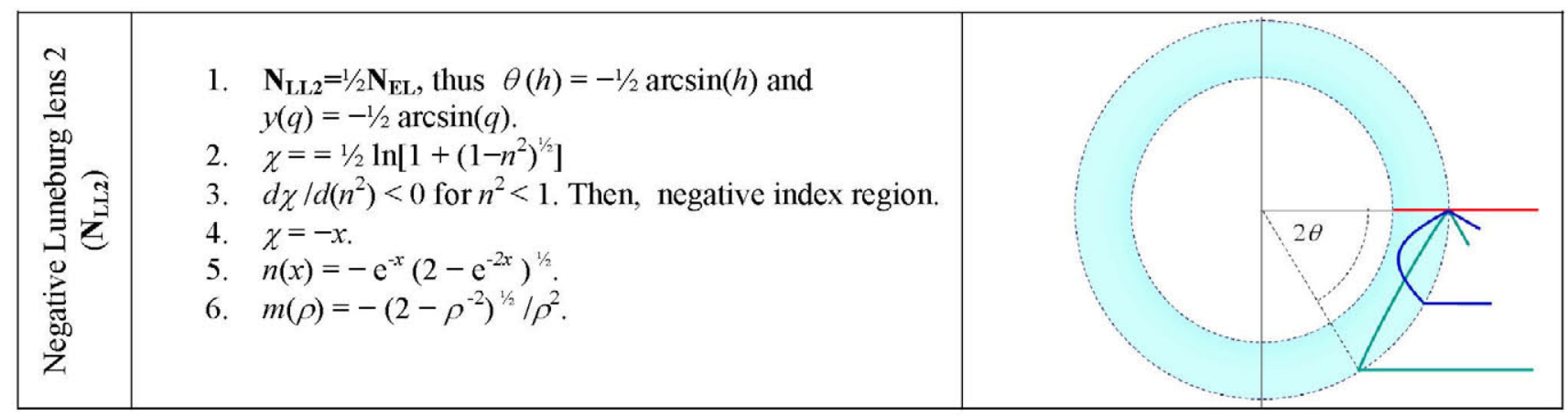

\section{TYPE B GRIN LENSES}

Consider now the case where $x_{\min }$ is a constant number, hence does not depend on $q$ (see Figure 2). This means that each ray trajectory passes through all the lens layers from $x_{\min }$ to $x_{\max }$ (which is fixed to 0 ).

The maximum value of $q\left(q_{\max }\right)$ must be smaller than the minimum value of $n^{2}\left(n_{\min }^{2}\right)$ so the rays can cross the entire refractive region to design. Then, according to Eq. (5) we have

$$
q_{\max }^{2}=1-s_{\min }<n_{\min }^{2}=1-t_{\max }
$$

thus, $t_{\max }<s_{\min }$. Since the function $y(q)$ is only defined between $-q_{\max }$ and $q_{\max }$, the variable $s$ must be in the range $s_{\min }$, 1. The integral from Eq. (7) now has a new form

$$
f(s)=\int_{0}^{t_{\max }} \frac{\dot{\chi}}{\sqrt{s-t}} d t
$$

where $t_{\text {max }}$ is fixed and

$$
f(s)=-\frac{y(\sqrt{1-s})}{\sqrt{1-s}}
$$

Eq. (17) is a Fredholm integral equation of the first kind where $\dot{\chi}$ is the unknown function and $y(q)=y\left((1-s)^{1 / 2}\right)$ is known [5][6]. This particular integral equation is sometimes called Abel integral equation with fixed limits.

According to [8] the solution of Eq. (17) is given by

$$
\chi_{t}(t)=-\frac{A}{\sqrt[4]{t}} \frac{d}{d t}\left[\int_{t}^{t_{\max }} \frac{\left.\int_{0}^{x} \frac{f(s)}{\sqrt[4]{s} \sqrt[4]{x-s}} d s\right]}{\sqrt[4]{x-t}} d x\right]
$$

The problem is that we do not know $f(s)$ for $s<s_{\min }$, so we can not apply Eq.(19). However, Eq. (17) can be sometimes solved numerically using the Singular Value Decomposition.

\subsection{Singular Value Decomposition}

For a given integral kernel $K$, the Singular Value Decomposition (in the further text SVD) exists if the kernel $K$ (which in our case is $\left.K(s, t)=1 /\left(s^{-} t\right)^{1 / 2}\right)$ is square integrable (as it is in our case) and takes the form

$$
K(s, t)=\sum_{i=1}^{\infty} \mu_{i} u_{i}(s) v_{i}(t)
$$

Where the functions $u_{\mathrm{i}}$ and $v_{\mathrm{i}}$ are called the left and the right singular functions, and $\mu_{\mathrm{i}}$ are called the singular values [7].

For numerical calculation we need to do a discretization of Eq. (17) as 


$$
f(s)=\sum_{j=1}^{j_{\operatorname{maz}}}\left(\int_{\left(\Delta_{j}-1\right) j}^{\Delta_{j} j} \frac{\dot{\chi}}{\sqrt{s-t}} d t\right)
$$

where $\Delta_{\mathrm{j}}=t_{\max } / j_{\max }, j_{\max }$ being the number of terms in the sum of Eq. (21). The integrals from Eq. (21) can be calculated as

$$
f(s)=\sum_{j=1}^{j_{\max }}\left(\int_{\left(\Delta_{j}-1\right) j}^{\Delta_{j} j} \frac{\dot{\chi}}{\sqrt{s-t}} d t\right) \approx 2 \sum_{j=1}^{j_{\max }} \dot{\chi}\left(\Delta_{j}(j-0.5)\right)\left(\sqrt{s-\Delta_{j}(j-1)}-\sqrt{s-\Delta_{j} j}\right)
$$

Now we sample values of $s$ so Eq. (22) is given as

$$
f\left(\Delta_{i}(i-0.5)\right)=2 \sum_{j=1}^{j_{\max }} \dot{\chi}\left(\Delta_{j}(j-0.5)\right)\left(\sqrt{\Delta_{i}(i-0.5)-\Delta_{j}(j-1)}-\sqrt{\Delta_{i}(i-0.5)-\Delta_{j} j}\right)=\sum_{j=1}^{j_{\max }} \dot{\chi}\left(\Delta_{j}(j-0.5)\right) L(i, j)
$$

where $\Delta_{\mathrm{i}}=\left(s_{\max }-s_{\min }\right) /\left(i_{\max }-i_{\min }+1\right),\left(i_{\max }-i_{\min }+1\right)$ being the number of samples taken between $s_{\min }$ and $s_{\max }$. The minimum value of $i$ in Eq. (23) is such that $\left(i_{\min }-1\right) \Delta_{\mathrm{i}}<s_{\min }<i_{\min } \Delta_{\mathrm{i}}$. Since $t_{\max }<s_{\min }$ then $\Delta_{\mathrm{j}} j_{\max }<i_{\min } \Delta_{\mathrm{i}}$.

Using matrix and vector notation we have discrete form of Eq. (17)

$$
\left(\begin{array}{l}
f_{i_{\min }} \\
\ldots \\
f_{i} \\
\ldots \\
f_{i_{\max }}
\end{array}\right)=\left(\begin{array}{lllll}
L\left(i_{\min }, 1\right) & \ldots & L\left(i_{\min }, j\right) & \ldots & L\left(i_{\min }, j_{\max }\right) \\
\ldots & \ldots & \ldots & \ldots & \ldots \\
L(i, 1) & \ldots & L(i, j) & \ldots & L\left(i_{,} j_{\max }\right) \\
\ldots & \ldots & \ldots & \ldots & \ldots \\
L\left(i_{\max }, 1\right) & \ldots & L\left(i_{\max }, j\right) & \ldots & L\left(i_{\max }, j_{\max }\right)
\end{array}\right)\left(\begin{array}{l}
\dot{\chi}_{1} \\
\ldots \\
\dot{\chi}_{j} \\
\ldots \\
\dot{\chi}_{j_{\max }}
\end{array}\right)
$$

Using discrete form of the SVD the matrix T from Eq. (24) takes the following form [7]

$$
\mathrm{T}=\mathrm{U} \Sigma \mathrm{V}^{\mathrm{T}}
$$

Here, $\Sigma$ is a diagonal matrix satisfying: $\Sigma=\operatorname{diag}\left(\sigma_{1}, \sigma_{2}, \ldots \sigma_{\mathrm{n}}\right)$, and $\sigma_{1} \geq \sigma_{2} \geq \ldots \geq \sigma_{\mathrm{n}} \geq 0$. The matrices $\mathrm{U}$ and $\mathrm{V}$ consist of the left and right singular vectors, i.e. $U=\left(\mathbf{u}_{1}, \mathbf{u}_{2}, \ldots, \mathbf{u}_{\mathbf{n}}\right)$ and $V=\left(\mathbf{v}_{1}, \mathbf{v}_{2}, \ldots, \mathbf{v}_{\mathbf{n}}\right)$.

As an example, let's set $t_{\max }=0.5, s_{\max }=1, s_{\min }=0.5$. Figure 3 show the left vectors for the kernel defined in Eq. (17). The right vectors have similar form, $\mathbf{v}_{\mathrm{i}}[l]=\mathbf{u}_{\mathrm{i}}[n-l+1], l$ being a number $1 \leq l \leq n$.

According to [7] the solution of Eq.(24) is given as

$$
\dot{\chi}=\mathrm{T}^{-1} \mathbf{f}=\sum_{i=1}^{n} \frac{\mathbf{u}_{i}^{T} \mathbf{f}}{\sigma_{i}} \mathbf{v}_{i}
$$

However, this solution is called "naive" since there are extremely large errors coming from the noisy components related to the smaller singular values. When the Picard condition [7] is fulfilled, this noise can be decreased by truncating the sum in Eq. (26). The solution of Eq. (26) is obtained by retaining the first $k$ components of the "naive" solution, $k$ being the number such that for all $i \leq k$ the corresponding coefficients $\left|\mathbf{u}_{i}^{T} \mathbf{f}\right|$, on average, decay faster than the $\sigma_{i}$.

$$
\dot{\boldsymbol{\chi}}_{k}=\sum_{i=1}^{k} \frac{\mathbf{u}_{i}^{T} \mathbf{f}}{\sigma_{i}} \mathbf{v}_{i}
$$



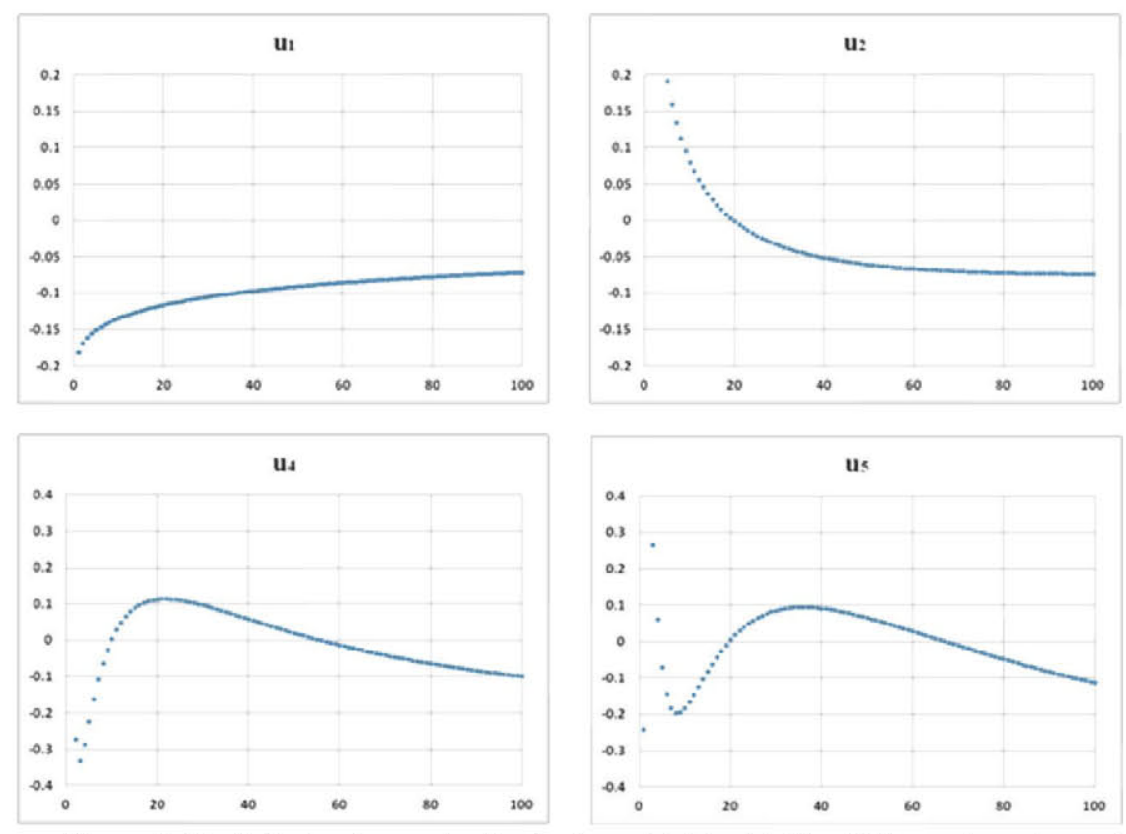

Figure 3 The left singular vector for the kernel defined in Eq. (17), number of samples is $n=100$.

\subsection{Examples of Type B GRIN lens}

In order to explain the SVD method, let consider first the next problem, called Problem 1. For Eq. (17) let define the function $f(s)$ as

$$
f(s)=\frac{\left(4 s^{\frac{3}{2}}\right)}{3}-\frac{2 \sqrt{s-t_{\max }}\left(2 s+t_{\max }\right)}{3}
$$

where $t_{\max }=0.5$. The true solution of this problem is known, $\dot{\chi}(t)=t$. Let calculate this solution using the SVD method. After repeating the calculus given by Eq. (22)-(24) and doing the SVD for the matrix T, one gets the Picard plots as presented in Figure 4.
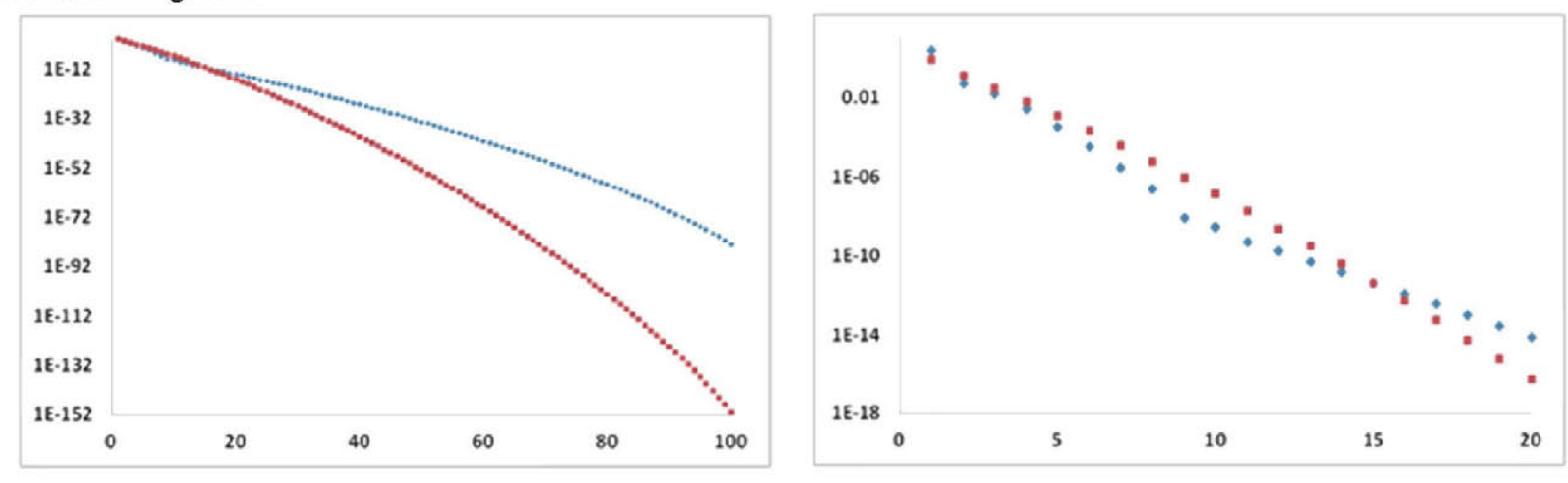

Figure 4 The Picard plot for Problem 1. The values of $\left|\mathbf{u}_{i}^{T} \mathbf{f}\right|$ are presented in blue and the $\sigma_{i}$ are presented in red (left).

Close up of the same Picard plot (right)

According to the graphs presented in Figure 4, it is clear that the coefficients $\left|\mathbf{u}_{i}^{T} \mathbf{f}\right|$ decay faster than the $\sigma_{i}$ if $i \leq 9$. If we put $k=9$ in Eq. (27) we get a solution very close to the true one (see Figure 5). However, if we add more components, e.g. we put $k=14$, the solution start to oscillate introducing the noise as shown in Figure 5 . 

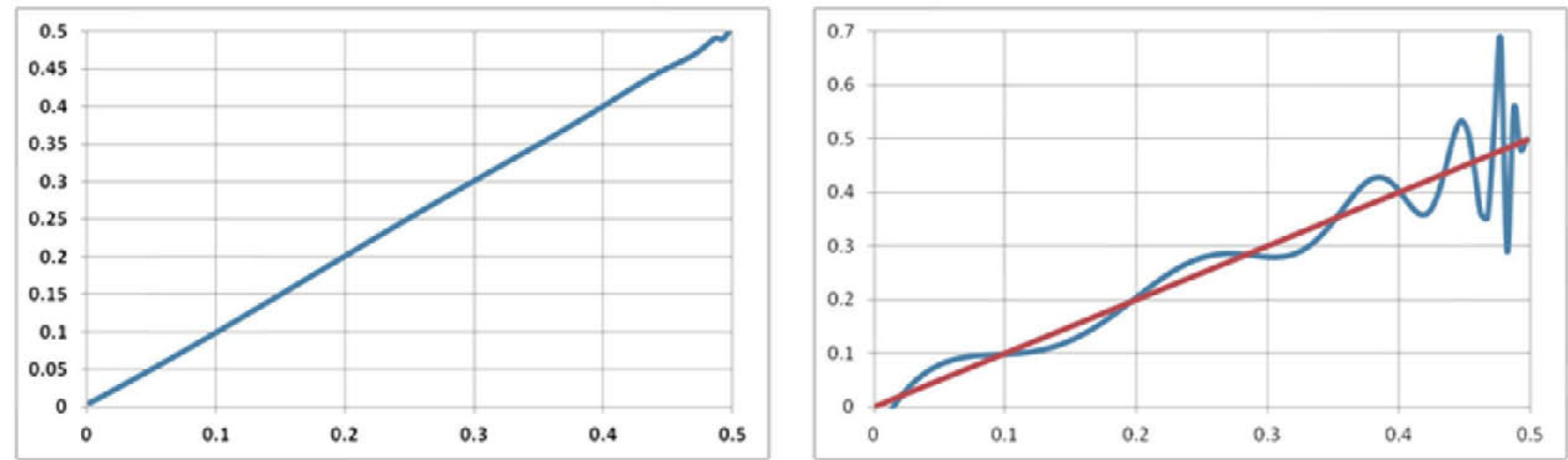

Figure 5 Solution of Problem 1 when $k=9$ (left). Solution of Problem 1 when $k=14$ (in blue) and true solution (in red), right

Consider now the following example (Figure 6), we will call it Problem 2. The angle of flight is given as

$$
y(q)=\arcsin (q)
$$

Eq. (7) applies with a change of sign (the problem is defined for $x \geq 0$ ), thus

$$
f(s)=\frac{\arcsin (\sqrt{1-s})}{\sqrt{1-s}}
$$

Assume that we have normalized the refractive index distribution so $n^{2}\left(0^{+}\right)=1$. Since we have previously ordered the refractive index distribution so $\mathrm{d}\left(n^{2}\right) / \mathrm{d} x \leq 0$, then we know that $0 \leq n^{2}(x) \leq 1$. Consequently (see Eq. (5)) $0 \leq t \leq 1$. Assume that $t_{\max }$ in Eq. (17) is again 0.5. Then our problem can be written as

$$
f(s)=\frac{\arcsin (\sqrt{1-s})}{\sqrt{1-s}}=\int_{0}^{0.5} \frac{\dot{\chi}}{\sqrt{s-t}} d t
$$
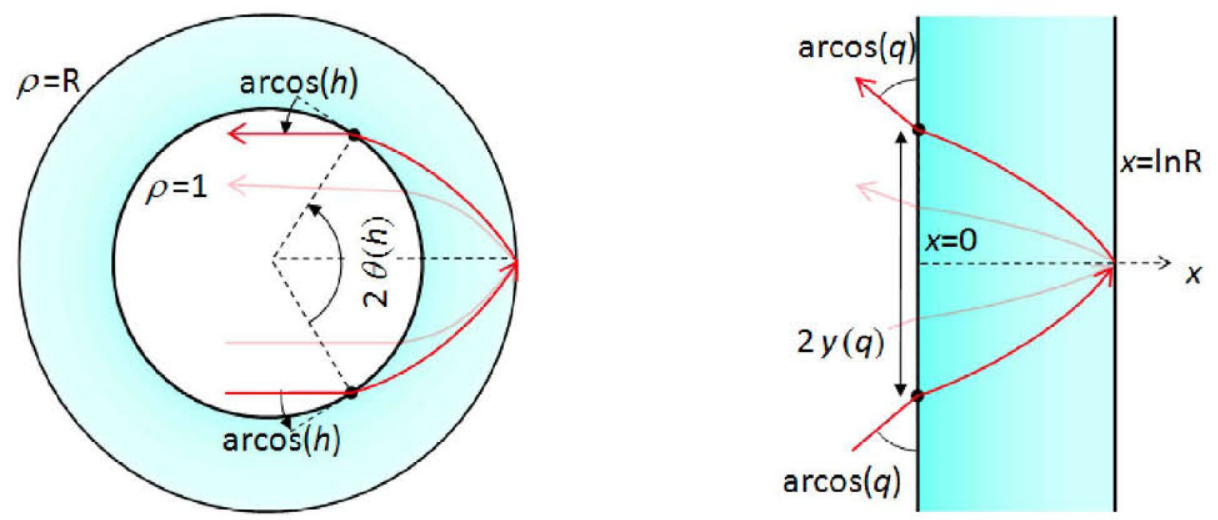

Figure 6 Problem 2: Rays flying parallel in the circle $\rho \leq 1$ are focussed at point with $\rho=\mathrm{R}$ (left), and its linear counterpart (right). $h=\sin \theta \Rightarrow \theta(h)=\arcsin (h)$

Figure 7 shows the Picard plot for this problem. According to these graphs, the coefficients $\left|\mathbf{u}_{i}^{T} \mathbf{f}\right|$ decay slower than the $\sigma_{i}$ for every $i$. This means that we can not truncate our solution and filter it from the noise, as we have done in Problem 1, hence Problem 2 cannot be solved with this SVD technique (see also references [5][7]).

Problem 2 arises when trying to substitute the refractive index distribution of the $\mathrm{M}$ lens by another distribution combined with an spherical mirror [11], so the angle of flight is the same. The aim is to find a refractive index 
distribution that avoids the need of refractive indices going to zero, as it happens in the $\mathrm{M}$ lens when $\rho=2$. Tyc et al give an approximate solution to this problem in [11]. Tyc at al also prove that there is no exact solution with positive refractive index distribution in ref. [12].
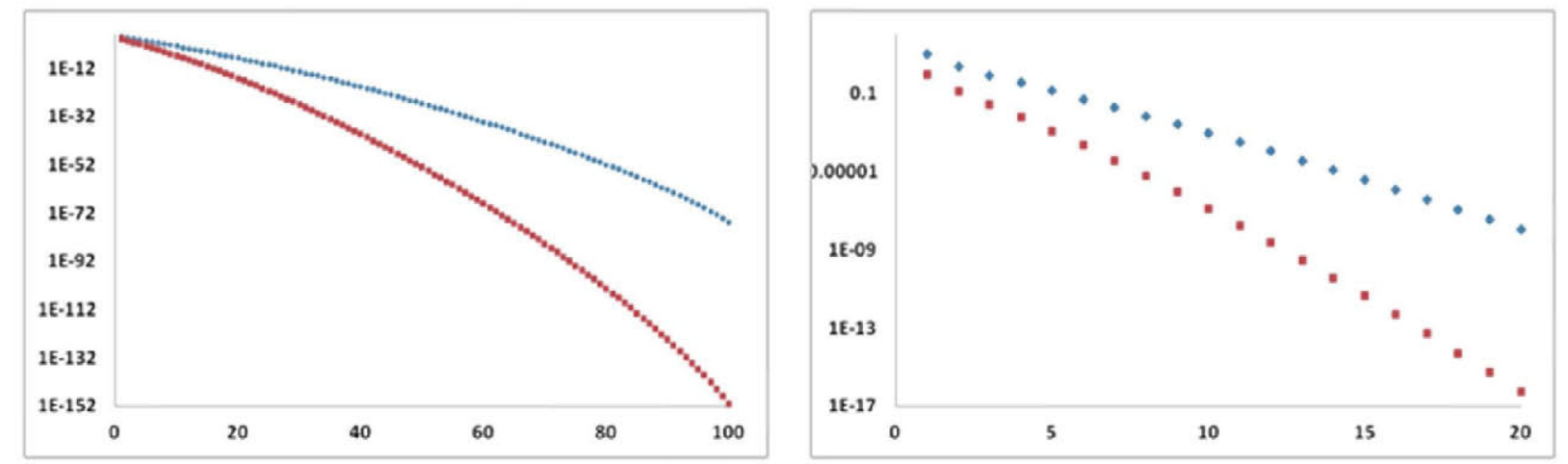

Figure 7 The Picard plot for Problem 2. The values of $\left|\mathbf{u}_{i}^{T} \mathbf{f}\right|$ are presented in blue and the $\sigma_{i}$ are presented in red (left). Close up of the same Picard plot (right)

\section{CONCLUSIONS}

In this paper the GRIN lenses design is organized in a new way. The GRIN lenses are divided into two groups, named Type A GRIN lenses, which are defined by the Abel integral equation, and Type B GRIN lenses (defined by the Abel integral equation with fixed limits). Due to convenience, both types are analyzed in a linear transformed space given by Eq. (1). For Type A GRIN lenses, we show how old and new refractive index distributions can be obtained with linear combinations of existing ones.

Also we present a novel numerical design method for Type B GRIN lenses based on the Singular Value Decomposition.

\section{ACKNOWLEDGEMENTS}

The authors would like to thank the Spanish Ministry MICINN (ENGINEERING METAMATERIALS: CSD200800066, DEFFIO: TEC2008-03773, SIGMASOLES: PSS-440000-2009-30, PERIMAGE: TEC2011-24019, TEC201016948, INNPACTO SIGMAMODULOS: INN110935C37B), MITYC (ECOLUX: TSI-020100-2010-1131, SEM: TSI020302-2010-65), the Madrid Regional Government (SPIR: 50/2010O.23/12/09,TIC2010 and O-PRO: PIE/209/2010) and UPM (Q090935C59) for the support given in the preparation of the present work.

\section{REFERENCES}

[1] Leonhardt, U., "Perfect imaging without negative refraction," New J. Phys. 11, 093040 (2009)

[2] Pendry, J. B., " Negative Refraction makes a Perfect Lens", Phy. Review Let. 85, 3966-3989 (200)

[3] Luneburg, R. K., [Mathematical Theory of Optics], University of California Press, Los Angeles (1964)

[4] Cornbleet, S., [Microwave and Geometrical Optics], Academic (1994)

[5] Groetsch, C. W., "Integral equations of the first kind, inverse problems and regularization: a crash course," J. Phys.: Conf. Ser. 73012001 (2007)

[6] Hermann, R., [Fractional calculus], World Scientific, Singapore (2011)

[7] Hansen, P. C., [Discrete Inverse Problems: Insight and Algorithms], SIAM (2010)

[8] Polyanin, A. D, and Manzhirov, A. V., [Handbook of Integral Equations], CRC Press, Boca Raton (1998)

[9] Kreyszig, E., [Differential Geometry], Dover, New York (1991)

[10]Miñano, J. C., Benítez, P., Santamaría, A., "Hamilton-Jacobi equation in momentum space", Optics Express 14, 9083-9092 (2006)

[11] Tyc, T., Sarbort, M., "Approximate magnifying perfect lens with positive refraction," arXiv:1010.3178v2 (2010)

[12]Tyc, T.,Herzanova, L., Sarbort, M., Bering, K., "Absolute instruments and perfect imaging in geometrical optics," New J. Phys. 13, 115004 (2011) 